\title{
Characterization of $\mathrm{Er}^{3+}: \mathrm{YVO}_{4}$ for microwave to optical transduction
}

\author{
Tian Xie, ${ }^{1,2}$ Jake Rochman, ${ }^{1,2}$ John G. Bartholomew, ${ }^{1,2,{ }^{*}}$ Andrei Ruskuc $\odot,{ }^{1,2}$ Jonathan M. Kindem,,${ }^{1,2,}$ Ioana Craiciu, $, 2, \ddagger$ \\ Charles W. Thiel, ${ }^{3}$ Rufus L. Cone, ${ }^{3}$ and Andrei Faraon ${ }^{1,2, \S}$ \\ ${ }^{1}$ Kavli Nanoscience Institute and Thomas J. Watson, Sr., Laboratory of Applied Physics, California Institute of Technology, \\ Pasadena, California 91125, USA \\ ${ }^{2}$ Institute for Quantum Information and Matter, California Institute of Technology, Pasadena, California 91125, USA \\ ${ }^{3}$ Department of Physics, Montana State University, Bozeman, Montana 59717, USA
}

(Received 24 March 2021; revised 18 June 2021; accepted 13 July 2021; published 16 August 2021)

\begin{abstract}
Quantum transduction between microwave and optical frequencies is important for connecting superconducting quantum platforms within a quantum network. Ensembles of rare-earth ions are promising candidates to achieve this conversion due to their collective coherence properties at microwave and optical frequencies. Erbium ions are of particular interest because of their telecom wavelength optical transitions that are compatible with fiber communication networks and components. Here, we report the optical and electron spin properties of erbium-doped yttrium orthovanadate $\left(\mathrm{Er}^{3+}: \mathrm{YVO}_{4}\right)$, including high-resolution optical spectroscopy, electron paramagnetic resonance studies, and an initial demonstration of microwave to optical conversion of classical fields. The highly absorptive optical transitions and narrow ensemble linewidths make $\mathrm{Er}^{3+}: \mathrm{YVO}_{4}$ promising for magneto-optic quantum transduction.
\end{abstract}

DOI: 10.1103/PhysRevB.104.054111

\section{INTRODUCTION}

Advances in quantum technologies are emerging quickly, including demonstrations of quantum computation and simulation using superconducting qubits and atomic qubits [1,2], distribution of entangled optical photons over long distances $[3,4]$, and quantum memories based on solid state or atomic ensembles [5,6]. Incorporating the best technologies from different physical systems into a single network requires coherent transfer of quantum information between different operating regimes. One way to achieve this is to employ a quantum transducer. A primary example is to convert quantum states encoded in microwave photons to optical frequencies, which would enable distributed quantum computing schemes based on superconducting qubits or spin qubits [7]. Many physical systems have been proposed for microwave to optical (M2O) transduction [8,9], including optomechanical systems $[10,11]$, electro-optical systems [12,13], atomic ensembles $[14,15]$, and others $[16,17]$.

Among the atomic ensemble approaches, rare-earth ions (REIs) in solids are a promising platform for $\mathrm{M} 2 \mathrm{O}$ transduction applications [14,18-21]. REIs can be optically addressed

\footnotetext{
${ }^{*}$ Current address: Centre of Engineered Quantum Systems, School of Physics, The University of Sydney, Sydney, NSW 2006, Australia; The University of Sydney Nano Institute, The University of Sydney, NSW 2006, Australia.

${ }^{\dagger}$ Current address: JILA, University of Colorado and NIST, Boulder, CO, USA; Department of Physics, University of Colorado, Boulder, CO, USA; National Institute of Standards and Technology (NIST), Boulder, CO, USA.

${ }^{\ddagger}$ Current address: Jet Propulsion Laboratory, California Institute of Technology, Pasadena, California 91125, USA.

§faraon@caltech.edu
}

using their narrow $4 f-4 f$ transitions, while resonant electron spin, nuclear spin, or magnon transitions can be used in the microwave domain. Although the optical absorption of a single REI is relatively weak, ensembles of REIs can exhibit large optical depths due to their narrow inhomogeneities at cryogenic temperatures [22,23]. Additionally, REIs doped into crystals can possess long coherence lifetimes in both the optical and spin domain [24,25], which gives the possibility of a built-in memory incorporated with the transducer. Lastly, isotopes of REIs with nonzero nuclear spin can offer zero-field hyperfine structure with gigahertz splittings [20,26], which enables transduction without an external magnetic field.

One promising transduction protocol involves using a cavity-enhanced Raman scattering process with a three-level system [18]. In this scheme, high transduction efficiency can be achieved when the product of the optical and spin cooperativities is large, which requires ensembles possessing large transition strengths and narrow inhomogeneities in both optical and microwave domains. More specifically, in the limit of adiabatic driving and low efficiency, the transduction efficiency scales as $\zeta \equiv\left(\frac{d_{31} d_{22} \mu_{21} \rho}{\Delta_{o} \Delta_{\mu}}\right)^{2}[18]$, where $\rho$ is the number density of REIs, $d_{i j}\left(\mu_{i j}\right)$ is the optical (spin) dipole moment between levels $i$ and $j$ of the three-level system, as shown in Fig. 1, and $\Delta_{o(\mu)}$ is the detuning from the atomic resonance in the optical (microwave) domain, which is described by the ensemble inhomogeneous linewidth (e.g., three inhomogeneous linewidths away). All these parameters depend strongly on the specific material, ion, and transitions used; consequently, there is a strong dependence of the transduction efficiency on the REI and host crystal chosen as well as the actual inhomogeneous linewidths in both optical and spin domains that are obtained from the specific samples used. 
(a)

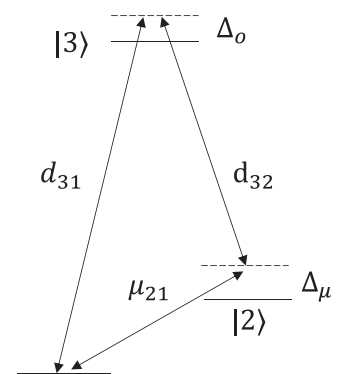

$|1\rangle$ (b)

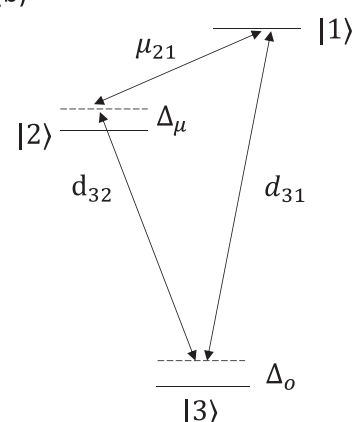

FIG. 1. Energy level diagram of a three-level system for the cavity-enhanced transduction process. (a) A $\Lambda$ system and (b) a $V$ system. $d_{i j}\left(\mu_{i j}\right)$ is the optical (spin) dipole moment between level $i$ and $j, \Delta_{o(\mu)}$ is the detuning from the atomic resonance in optical (microwave) domain.

Among the REIs, erbium is an attractive choice because its ${ }^{4} \mathrm{I}_{15 / 2}-{ }^{4} \mathrm{I}_{13 / 2}$ optical transitions occur in the lowest-loss telecommunication band for fiber-based optical communication networks. Erbium has been extensively studied for quantum information technologies in host crystals including $\mathrm{Y}_{2} \mathrm{SiO}_{5}$ [27-29], and there has been recent work in other hosts such as $\mathrm{YPO}_{4}$ [30], $\mathrm{Y}_{2} \mathrm{O}_{3}$ [31], $\mathrm{LiYF}_{4}$ [32], $\mathrm{LiNbO}_{3}$ [33], and $\mathrm{TiO}_{2}$ [34]. Yttrium orthovanadate $\left(\mathrm{YVO}_{4}\right)$ is an interesting host crystal because of its high site symmetry which leads to first-order insensitivity of optical transition frequencies to electric field, strong transition selection rules and wave function symmetries, and can readily be grown as high-quality crystals that exhibit exceptionally narrow inhomogeneous lines even at relatively high doping concentrations [23]. In this paper, we investigate erbium-doped $\mathrm{YVO}_{4}\left(\mathrm{Er}^{3+}: \mathrm{YVO}_{4}\right)$ for its potential application in quantum transduction. Previous studies of $\mathrm{Er}^{3+}: \mathrm{YVO}_{4}$ have reported crystal-field analysis [35], lasing properties [36], and absorption and relaxation dynamics $[37,38]$ down to $4 \mathrm{~K}$ with low-frequency resolution ( $>100 \mathrm{GHz}$, equivalent to nanometer level). Recently, ${ }^{167} \mathrm{Er}^{3+}: \mathrm{YVO}_{4}$ coherent dynamics were investigated, demonstrating a 15-s ground state hyperfine lifetime, suggesting that ${ }^{167} \mathrm{Er}^{3+}: \mathrm{YVO}_{4}$ is a promising material for quantum memory applications [39].

Here, we present the optical and spin properties of the even (zero nuclear spin) erbium isotopes in $\mathrm{Er}^{3+}: \mathrm{YVO}_{4}$ at $1 \mathrm{~K}$. We characterize the optical transition strength, optical inhomogeneity, and the electronic $g$ tensors using high-resolution $(\sim 1$ $\mathrm{MHz}$ ) optical spectroscopy. We also measure the ground-state spin inhomogeneity using electron paramagnetic resonance (EPR) techniques. The highly absorptive optical transitions and narrow inhomogeneities make $\mathrm{Er}^{3+}: \mathrm{YVO}_{4}$ a promising material for REI quantum transducers that we explore further here through coherent M2O conversion of classical signals.

This paper is organized as follows: Sec. II provides details on the material properties, energy level structure, selection rules, and the spin Hamiltonian studied in this paper. Section III introduces the experimental setup. Section IV presents the experimental results including optical transmission spectroscopy, EPR measurements, and the M2O transduction measurements. (a)

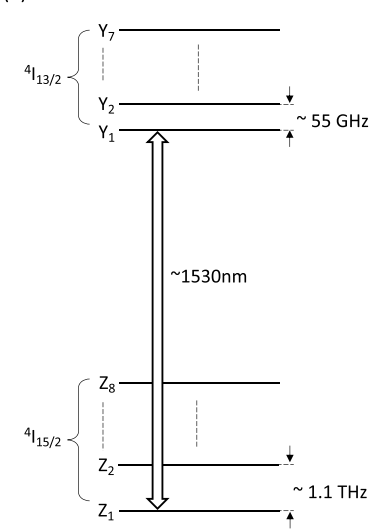

(b)

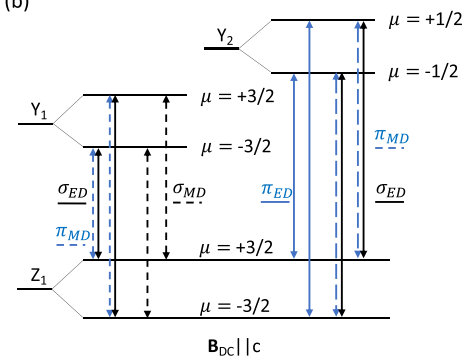

(c)

\begin{tabular}{|c|c|c|c|c|}
\hline$\mu$ & $-3 / 2$ & $+3 / 2$ & $-1 / 2$ & $+1 / 2$ \\
\hline$-3 / 2$ & $\sigma_{M D}$ & $\pi_{M D}, \bar{\sigma}_{E D}$ & $\left(\pi_{M D}, \sigma_{E D}\right.$ & $\pi_{E D}$ \\
\hline$+3 / 2$ & $\pi_{M D}, \sigma_{E D}$ & $\sigma_{M D}$ & $\pi_{E D}$ & $\pi_{M D}, \sigma_{E D}$ \\
\hline
\end{tabular}

FIG. 2. $\mathrm{Er}^{3+}: \mathrm{YVO}_{4}$ energy levels and selection rules. (a) The energy-level diagram of $\mathrm{Er}^{3+}: \mathrm{YVO}_{4}$ at zero applied magnetic field. (b) Partial diagram of $\mathrm{Er}^{3+}: \mathrm{YVO}_{4}$ crystal field and Zeeman levels with the crystal field quantum number and the selection rules under external magnetic field along the crystal symmetry $c$ axis $\left(\mathbf{B}_{\mathrm{DC}} \| c\right)$. $\pi$ and $\sigma$ indicate $\mathbf{E}_{\mathrm{AC}} \| c$ and $\mathbf{E}_{\mathrm{AC}} \| a$ polarization, respectively, with blue and black line styles. ED and MD denote the electric dipole and magnetic dipole transitions, with solid and dashed line styles, respectively. (c) The selection rules for ED and MD moment transitions in $D_{2 d}$ symmetry.

\section{BACKGROUND}

The host crystal $\mathrm{YVO}_{4}$ is a tetragonal crystal with the zircon structure and $D_{4 h}$ space group symmetry. The unitcell parameters are $a=b=7.1183 \AA$ and $c=6.2893 \AA$ [40]. There is one yttrium site in the unit cell with local $D_{2 d}$ point group symmetry, which can be substituted by an erbium ion without charge compensation. Due to the nonpolar symmetry at the substitutional site, there is no first-order DC Stark effect, which results in lower sensitivity to electric field noise that can cause decoherence and spectral diffusion of optical transitions [20]. The uniaxial site symmetry also greatly reduces the parameters needed to characterize the system compared with some alternative host crystals with lower symmetry $\left(\mathrm{Y}_{2} \mathrm{SiO}_{5}\right.$, for example).

$\mathrm{The} \mathrm{Er}^{3+}$ ion has 11 electrons in the $4 f$ shell, which gives the first two spin-orbit-split multiplets as ${ }^{4} I_{15 / 2}$ and ${ }^{4} I_{13 / 2}$, from the free ion Hamiltonian [41]. The crystal field with $D_{2 d}$ symmetry further splits each multiplet, characterized by the total angular momentum $J$, into $J+\frac{1}{2}$ Kramers doublets. Consequently, the ${ }^{4} \mathrm{I}_{15 / 2}\left({ }^{4} \mathrm{I}_{13 / 2}\right)$ level splits into 8 (7) levels, labeled as $Z_{1}$ to $Z_{8}\left(Y_{1}\right.$ to $\left.Y_{7}\right)$ in order from the lowest to the highest energy, as shown in Fig. 2(a). These labels transform to the irreducible representations $\Gamma_{6}$ or $\Gamma_{7}$ and can be described by the crystal field quantum number $\mu= \pm \frac{1}{2}, \pm \frac{3}{2}$ according to the $D_{2 d}$ point symmetry double group [42,43]. From the irreducible representations, the electric (ED) and magnetic dipole (MD) transition selection rules can be determined [32], as shown in Figs. 2(b) and 2(c).

For a small applied magnetic field $\mathbf{B}$, the Kramers doublets can be approximately described using an isomorphic effective spin Hamiltonian with $S=\frac{1}{2}$ [41]:

$$
\mathcal{H}_{\mathrm{eff}}=\mu_{B} \mathbf{B} \cdot \mathbf{g} \cdot \mathbf{S},
$$


where $\mathbf{g}$ is the electronic Zeeman tensor, and $\mathbf{S}$ is the effective spin- $\frac{1}{2}$ operator. Because of the local $D_{2 d}$ symmetry, only the diagonal terms of the $\mathbf{g}$ tensor are nonzero, which we label $g_{\|}$and $g_{\perp}$ for components parallel and perpendicular to the crystal symmetry $c$ axis, respectively.

At cryogenic temperature, only the $\mathrm{Z}_{1}$ level is populated because the crystal field splitting in the ground state is $\sim 1.1$ $\mathrm{THz}$ that is much larger than the available thermal energy $\left(\hbar \omega / k_{b} \approx 50 \mathrm{~K} \gg 1 \mathrm{~K}\right)$. The splitting between $\mathrm{Y}_{1}$ and $\mathrm{Y}_{2}$ is only $55 \mathrm{GHz}$ at zero field, which may be detrimental for coherent optical properties of $Y_{1}$ and $Y_{2}$ at high temperature (e.g., $>4 \mathrm{~K}$ ) due to resonant phonon absorption and emission [44], but should not be a concern for quantum transduction experiments that operate at much lower temperatures of $T$ $<100 \mathrm{mK}$. The following experiments and discussions are focused on the levels $Z_{1}, Y_{1}$, and $Y_{2}$. The optical transitions between these levels occur at $\sim 1530 \mathrm{~nm}$.

\section{EXPERIMENTAL SETUP}

Samples were cut and polished from a boule of $\mathrm{YVO}_{4}$ grown by Gamdan Optics and doped with a natural abundance of $\mathrm{Er}^{3+}$ isotopes, which is comprised of $77 \%$ nuclear-spinzero even isotopes and $23 \%$ of the odd isotope ${ }^{167} \mathrm{Er}^{3+}$ that has nuclear spin- $\frac{7}{2}$. The even-isotope $\mathrm{Er}^{3+}$ concentration was $140 \mathrm{ppm}$ (measured by secondary ion mass spectrometry). For the main experiments, we used a 200$\mu$ m-thick a-cut crystal mounted within a $2.4 \mathrm{GHz}$ loop-gap microwave resonator $(Q=860)$ machined from oxygen-free high-conductivity copper and mounted on the still plate (base temperature of $1 \mathrm{~K}$ ) of a dilution refrigerator, as shown in Fig. 3. The loop-gap resonator was mounted within a fiber coupled U-bench (Thorlabs FBC-1550-APC) with two fiber collimators (Thorlabs PAF-X-2-C) for optical transmission measurements. The total optical coupling efficiency through the U-bench setup was $35 \%$ at $1 \mathrm{~K}$, limited by misalignment due to differential thermal contraction during cooldown. The light propagation direction was along the $a$ axis of the crystal, which allowed measurement of both $\pi$ and $\sigma$ polarized spectra. A DC magnetic field ( $\mathbf{B}_{\mathrm{DC}}$ ) was applied to the crystal along the $c$ axis using a home-built split-coil superconducting magnet that generated fields of up to $120 \mathrm{mT}$. To determine the electronic Zeeman $g$ tensors for levels $Z_{1}, Y_{1}$, and $Y_{2}$, we placed the crystal within a two-axis superconducting magnet to perform magnetic field rotations with respect to the crystal symmetry axes (not shown in the Fig. 3 schematic).

Optical measurements are illustrated in the lower half of the diagram in Fig. 3. We used a tunable diode laser (Toptica CTL) as our light source. The absolute laser frequency was calibrated using a wavemeter (Bristol 671A). The laser light was split into two paths. The signal path, containing $10 \%$ of the power, was passed through a polarization controller (POL) and a fiber-coupled acousto-optic modulator (AOM) to perform pulsed measurements. The $90 \%$ path was used as the local oscillator for heterodyne measurements. For continuous wave optical measurements, the light transmitted through the crystal was measured with a photodiode. For pulsed lifetime measurements, another fiber-coupled AOM was used as a shutter to gate a superconducting nanowire single-photon detector (SNSPD) that detected the fluorescence signal. Mi-

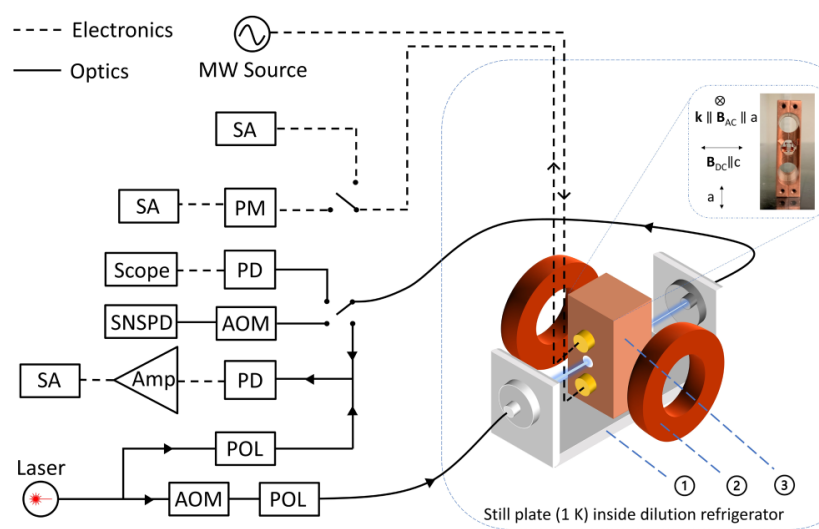

FIG. 3. Experimental setup inside and outside the dilution fridge for optical and microwave measurements. (1): fiber-to-fiber U-bench, (2): superconducting magnet, (3): loop-gap microwave resonator, AOM: acousto-optic modulator, POL: polarization controller, PD: photodiode, SNSPD: superconducting nanowire single-photon detector, Amp: amplifier, SA: spectrum analyzer, PM: power meter, MW: microwave. Solid lines show the optical path, and dashed lines indicate electronic connections. Inset: A front view of an a-cut sample sitting in the loop-gap resonator. The light propagates along the crystal $a$ axis, which allows $\pi$ and $\sigma$ excitation. $\mathbf{B}_{\mathrm{DC}}$ is along the crystal $c$ axis. The direction of the oscillating magnetic field $\left(\mathbf{B}_{\mathrm{AC}}\right)$ generated by the loop-gap resonator is along the crystal $a$ axis.

crowave measurements are illustrated in the upper half of the diagram in Fig. 3. The microwave signal was coupled into and out of the loop-gap resonator using two antennas positioned within the loops [45]. For EPR measurements, we used a function generator (TPI-1002-A) to generate a $100 \mathrm{kHz}$ frequency-modulated (FM) microwave signal centered at the loop-gap resonator frequency $(2.4 \mathrm{GHz})$. A radiofrequency (RF) power meter (RF Bay RPD-5513) was used to detect and mix down the FM signal that was transmitted through the antennas. The beat-note amplitude was measured using a spectrum analyzer (FieldFox N9915A). For the M2O transduction experiment, a microwave tone was generated from the spectrum analyzer, and a laser tone was sent to the crystal. For detection, we combined the collected light with the local oscillator path and detected the interference on a fast photodiode (UPD-35-IR2-FC). The resulting heterodyne signal was amplified (WBA2080-35A) and recorded using the spectrum analyzer.

\section{EXPERIMENTAL RESULTS}

\section{A. Optical transmission spectrum}

High-resolution optical transmission spectra for $Z_{1}-Y_{1}$ and $Z_{1}-Y_{2}$ were performed as a function of the applied bias magnetic field strength for $\mathbf{B}_{\mathrm{DC}} \| c$ [see Figs. 4(a)4(d)]. We observed narrow inhomogeneously broadened and highly absorptive transition lines. For $\mathrm{Z}_{1}-\mathrm{Y}_{1}$ under $\sigma$ polarized excitation, there were four well-resolved transitions with an average inhomogeneous linewidth of $184 \pm 10 \mathrm{MHz}$, as shown in Fig. 4(a). The number of allowed transitions was consistent with the theoretical calculation [see Fig. 2(b)]. For $\mathrm{Z}_{1}-\mathrm{Y}_{2}$ with $\pi$ polarized excitation [Fig. 4(b)], we observed four highly absorbing transitions with an average inhomoge- 
(a)

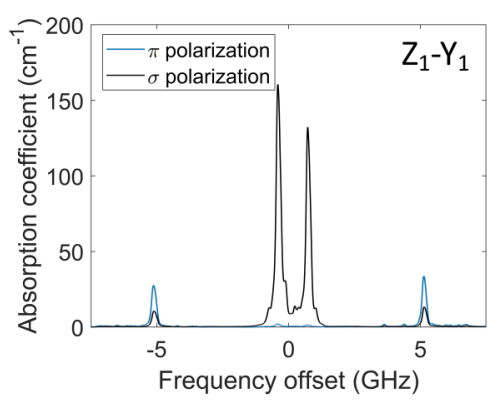

(b)

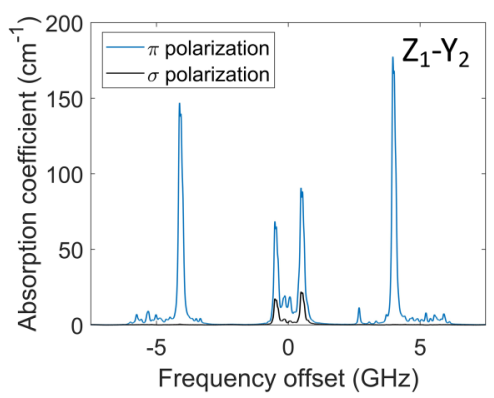

(c)

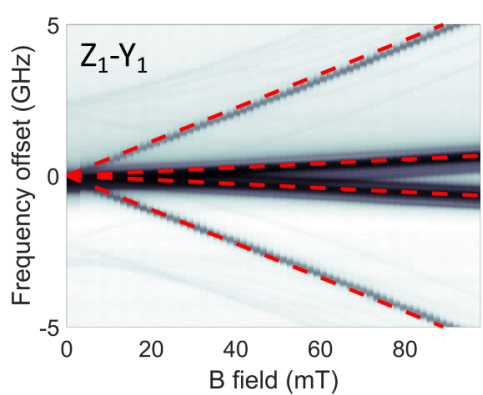

(d)

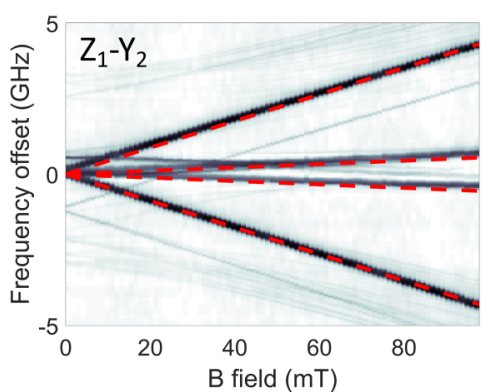

(e)

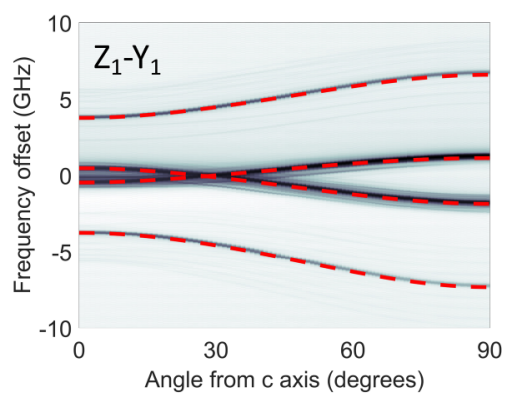

(f)

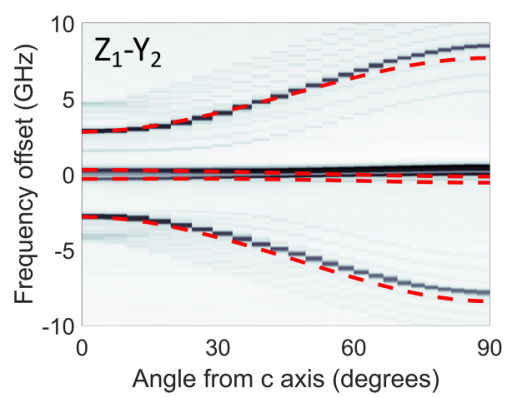

FIG. 4. The high-resolution optical transmission spectrum of $140 \mathrm{ppm} \mathrm{Er}^{3+}: \mathrm{YVO}_{4}$. (a) and (b) The optical transmission spectrum of $\mathrm{Z}_{1}-\mathrm{Y}_{1}$ and $\mathrm{Z}_{1}-\mathrm{Y}_{2}$, respectively, at an applied field of $90 \mathrm{mT}$ at $1 \mathrm{~K}$. The blue line labels $\pi$ polarization, and the black line labels $\sigma$ polarization. (c) and (d) The magnetic field ramp of $\mathrm{Z}_{1}-\mathrm{Y}_{1}$ and $\mathrm{Z}_{1}-\mathrm{Y}_{2}$ transmission spectra with $\sigma$ and $\pi$ polarization, respectively. The red dashed lines are the fitting from the effective spin Hamiltonian. (e) and (f) The transmission spectra as a magnetic field of $75 \mathrm{mT}$ rotated from parallel to the crystal $c$ axis to parallel with crystal $a$ axis. The red dashed lines are the fitting from the effective spin Hamiltonian.

neous linewidth of $163 \pm 14 \mathrm{MHz}$. The ratio of the absorption strength from the same dipole moments connecting different ground-state spin levels gave the thermal distribution between ground-state spin levels, which was consistent with a $1 \mathrm{~K}$ crystal temperature. Additional absorption lines were observed from the ${ }^{167} \mathrm{Er}$ isotope (40 ppm). These spectrally resolved optical transitions allowed us to individually address transitions between different Zeeman levels. Also, since all four transitions are allowed, this can provide a suitable three-level system for transduction [18], as described in the Introduction section.

From the total integrated absorption coefficient, we calculated the fractional ED and MD absorption oscillator strengths $f_{i j, q}$ contributing to each polarized absorption using [23,25]

$$
\begin{aligned}
f_{i j, q}^{\mathrm{ED}} & =4 \pi \epsilon_{0} \frac{m_{e} c}{\pi e^{2}} \frac{1}{N} \frac{n_{q}}{\chi_{L}} \int \alpha_{q}^{\mathrm{ED}}(v) d \nu, \\
f_{i j, q}^{\mathrm{MD}} & =4 \pi \epsilon_{0} \frac{m_{e} c}{\pi e^{2}} \frac{1}{N} \frac{1}{n_{q}} \int \alpha_{q}^{\mathrm{MD}}(v) d \nu,
\end{aligned}
$$

where $\epsilon_{0}$ is the vacuum permittivity, $m_{e}$ is the electron mass, $c$ is the speed of the light, $e$ is the charge of an electron, $N$ is the number density, $q$ is the optical polarization state, $n_{q}$ is the refractive index experience by polarization $q, \chi_{L}=\left(\frac{n_{q}^{2}+2}{3}\right)^{2}$ is the local electric field correction factor (virtual cavity model), and $\alpha_{q}$ is the absorption coefficient for different polarizations. Note that the total transition oscillator strength $f_{i j}$ is given by summing the fractional oscillator strengths $f_{i j, q}$ over the three possible orthogonal polarization states $q\left(f_{i j}=\sum_{q} f_{i j, q}\right)$. Because the levels are not degenerate when split by the Zeeman effect, the emission oscillator strength is the same as the absorption oscillator strength $f_{j i}=f_{i j}$. For $\mathrm{YVO}_{4}$, the refractive index along the $c(a)$ axis at this wavelength is 2.15 (1.95) [46]. With the doping concentration equal to $140 \mathrm{ppm}$, the even-isotope erbium dopant number density is $N=1.75 \times 10^{18}$ ions $/ \mathrm{cm}^{3}$, distributed between the two Zeeman levels. The corresponding effective dipole moment was calculated from the oscillator strength using $d_{i j, q}^{2}=\frac{\hbar e^{2}}{2 m \omega} f_{i j, q}$. The corresponding contributions to the radiative lifetime were calculated using $[23,25]$

$$
\begin{aligned}
\frac{1}{\tau_{\mathrm{rad}, q}^{\mathrm{ED}}} & =\frac{2 \pi e^{2}}{\epsilon_{0} m_{e} c} \frac{\chi_{L} n_{q}}{\lambda_{0}^{2}} \frac{f_{j i, q}^{\mathrm{ED}}}{3}, \\
\frac{1}{\tau_{\mathrm{rad}, q}^{\mathrm{MD}}} & =\frac{2 \pi e^{2}}{\epsilon_{0} m_{e} c} \frac{n_{q}^{3}}{\lambda_{0}^{2}} \frac{f_{j i, q}^{\mathrm{MD}}}{3},
\end{aligned}
$$

where $\lambda_{0}$ is the transition wavelength in vacuum. The calculated integrated absorption coefficient, oscillator strength, radiative lifetime, and dipole moment for each transition are listed in Table I. From these numbers, we obtained the total oscillator strength as $f_{\mathrm{ED}}=1.7 \times 10^{-7}$ and $f_{\mathrm{MD}}=1.3 \times$ $10^{-6}$ for the $Z_{1}-Y_{1}$ transition, and $f_{\mathrm{ED}}=1 \times 10^{-6}$ and $f_{\mathrm{MD}}=1 \times 10^{-6}$ for the $\mathrm{Z}_{1}-\mathrm{Y}_{2}$ transition. The measured MD transition oscillator strength agreed with the theoretical calculation in Ref. [47] to within a factor of 2. The total oscillator strength of $\mathrm{Er}^{3+}: \mathrm{YVO}_{4}$ is relatively strong compared with other erbium-doped crystals, as summarized in Ref. [22], which are typically in the range of $f \approx 10^{-7}$ for the ${ }^{4} \mathrm{I}_{15 / 2}$ to ${ }^{4} \mathrm{I}_{13 / 2}$ transitions. From the calculated radiative lifetimes shown in Table I, we obtained the total radiative decay rate 
TABLE I. Optical properties of $\mathrm{Er}^{3+}: \mathrm{YVO}_{4}$ transitions including the transition wavelength, optical inhomogeneities, integrated absorption coefficients, oscillator strength, dipole moment, and the radiative lifetime of $\mathrm{Z}_{1}-\mathrm{Y}_{1}$ and $\mathrm{Z}_{1}-\mathrm{Y}_{2}$ transitions.

\begin{tabular}{|c|c|c|c|c|c|c|c|c|}
\hline \multirow[t]{3}{*}{$\mathrm{Z}_{1}-\mathrm{Y}_{1}$} & 1529.21 & $184 \pm 10$ & $\sigma$ & ED & 7.3 & 0.8 & 1 & 5.7 \\
\hline & & & & MD & 89.9 & 9.0 & 3.3 & 85 \\
\hline & & & $\pi$ & MD & 18.0 & 2.0 & 1.6 & 13.9 \\
\hline $\mathrm{Z}_{1}-\mathrm{Y}_{2}$ & & & & MD & 45.5 & 5.1 & 2.5 & 35.3 \\
\hline
\end{tabular}

of $\frac{1}{\tau_{\mathrm{rad}}}=\sum_{q}\left(\frac{1}{\tau_{\mathrm{rad}, q}^{\mathrm{ED}}}+\frac{1}{\tau_{\mathrm{rad}, q}^{\mathrm{MD}}}\right)$ to be $(8.1 \mathrm{~m}) \mathrm{s}^{-1}$ for $\mathrm{Y}_{1}-\mathrm{Z}_{1}$ and $(6.2 \mathrm{~ms})^{-1}$ for $\mathrm{Y}_{2}-\mathrm{Z}_{1}$ transitions.

To determine the electronic Zeeman $g$ factors, we measured the $\mathrm{Z}_{1}-\mathrm{Y}_{1}$ and $\mathrm{Z}_{1}-\mathrm{Y}_{2}$ optical transition frequencies as a function of the bias magnetic field $\mathbf{B}_{\mathrm{DC}}$, shown in Figs. 4(c) and 4(d). The red dashed lines were derived from an effective spin Hamiltonian fitted to the data. To determine $g_{\perp}$, we performed a magnetic field rotation experiment at a field strength of $\sim 75 \mathrm{mT}$, as shown in Figs. 4(e) and 4(f), where the field direction was rotated from the $c$ to the $a$ axis. With the knowledge of the $g$ factors for $Z_{1}$, we extracted the $g$ factors for $Y_{1}$ and $Y_{2}$ by fitting the data to an effective spin Hamiltonian model, as summarized in Table II. We attributed the deviation of the fitting results at $\sim 90^{\circ}$ in the $\mathrm{Z}_{1}-\mathrm{Y}_{2}$ rotation pattern to the quadratic Zeeman effect [49], which was not included in the model.

To investigate the decay branching ratio of the $\mathrm{Y}_{1}-\mathrm{Z}_{1}$ transition relative to relaxation to other crystal field levels (e.g., $Z_{2}$ to $Z_{8}$ and $Y_{2}$ ), defined as $\gamma_{Y_{1}-Z_{1}} / \sum_{L} \gamma_{Y_{1}-L}$, we measured the fluorescence decay lifetimes by exciting ensembles to $\mathrm{Y}_{1}$ or $\mathrm{Y}_{2}$ at $1 \mathrm{~K}$. To minimize the impact of radiation trapping [50], which is present at the center of the inhomogeneous line, we excited the ensemble with the laser frequency detuned by two inhomogeneous linewidths to higher frequency from the center of the relevant absorption peak. The fluorescence data detected on the SNSPD are shown in Fig. 5. When population was excited to $\mathrm{Y}_{1}\left(\mathrm{Y}_{2}\right)$, the extracted lifetime $\tau_{f}$ was $3.34 \pm 0.01 \mathrm{~ms}(3.30 \pm 0.01 \mathrm{~ms})$. The measured $\mathrm{Y}_{1}$ lifetime is longer than the reported $2.5 \mathrm{~ms}$ lifetime of ${ }^{4} \mathrm{I}_{13 / 2}$ level for a $0.1 \% \mathrm{Er}^{3+}: \mathrm{YVO}_{4}$ sample [35,51], which is reasonable considering the difference in sample concentration and the experimental spectral resolution. Given the $55 \mathrm{GHz}$ energy separation between $Y_{1}$ and $Y_{2}$, the phonon relaxation be-

TABLE II. The electronic Zeeman $g$ factors of $\mathrm{Er}^{3+}: \mathrm{YVO}_{4}$. The $g$ factors of the $Z_{1}$ level are from Ref. [48]. The $g$ factors of the $Y_{1}$ and $\mathrm{Y}_{2}$ levels are from fitting to experimental data using the effective spin Hamiltonian.

\begin{tabular}{lcc}
\hline \hline Levels & $\left|g_{\|}\right|$ & $\left|g_{\perp}\right|$ \\
\hline $\mathrm{Z}_{1}$ & 3.544 & 7.085 \\
$\mathrm{Y}_{1}$ & $4.51 \pm 0.02$ & $4.57 \pm 0.01$ \\
$\mathrm{Y}_{2}$ & $2.74 \pm 0.01$ & $6.74 \pm 0.15$ \\
\hline \hline
\end{tabular}

tween the states can significantly modify the branching ratios to $\mathrm{Z}_{1}$ as the temperature is varied. In the low-temperature limit $(\mathrm{kT} \ll \hbar \Delta)$, phonon absorption from $\mathrm{Y}_{1}$ to $\mathrm{Y}_{2}$ is suppressed, and the branching ratio for the $\mathrm{Y}_{1}-\mathrm{Z}_{1}$ transition is at least $\beta_{\mathrm{Y}_{1}}=\tau_{f_{-} \mathrm{Y}_{1}} / \tau_{\text {rad }} \mathrm{Y}_{1}=41.5 \%$. To specify the $\mathrm{Y}_{2}-\mathrm{Z}_{1}$ branching ratio, further experiments are needed to distinguish emission between $\mathrm{Y}_{1}-\mathrm{Z}_{1}$ and $\mathrm{Y}_{2}-\mathrm{Z}_{1}$ and determine the spontaneous phonon relaxation rate between $Y_{2}$ and $\mathrm{Y}_{1}$ as a function of temperature.

\section{B. EPR measurement}

Here, we study the spin inhomogeneity by measuring the EPR spectrum as a function of the applied magnetic field. We placed a 2 -mm-thick $(10 \times$ thicker than the optical measurements) sample into the loop-gap microwave resonator $\left(\mathbf{B}_{\mathrm{AC}} \| a\right)$. When the external magnetic-field-induced splitting $\left(\mathbf{B}_{\mathrm{DC}} \| c\right)$ causes the $\mathrm{Z}_{1}$ spin transition to be near the loop-gap resonance, the center frequency of the resonator is slightly shifted due to dispersive coupling with the spin ensemble. The ensemble coupling is expected to be $\Omega=\mu_{21} \sqrt{\frac{\rho \Delta n(T) \eta \omega_{o} \mu_{0}}{2 \hbar}}$, where $\mu_{21}$ is the spin magnetic dipole moment $\left(g_{\perp}\right.$ of the $Z_{1}$ level in this configuration), $\rho$ is the number density of the ions, $\Delta n(T)$ is the population difference between the spin levels, $\omega_{o}$ is the center frequency of the resonator, $\eta$ is the magnetic energy fraction in the $\mathrm{Er}^{3+}: \mathrm{YVO}_{4}$ crystal, $\hbar$ is the reduced

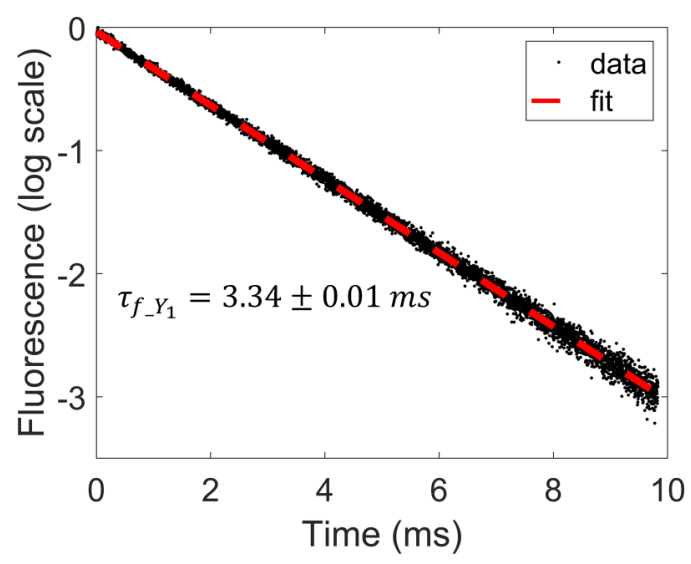

FIG. 5. $\mathrm{Y}_{1}$ lifetime measurement via fluorescence decay at $1 \mathrm{~K}$. Exponential fit is showed by the red dashed line, with decay constant $\tau_{f_{-} Y_{1}}=3.34 \pm 0.01 \mathrm{~ms}$. 
(a)

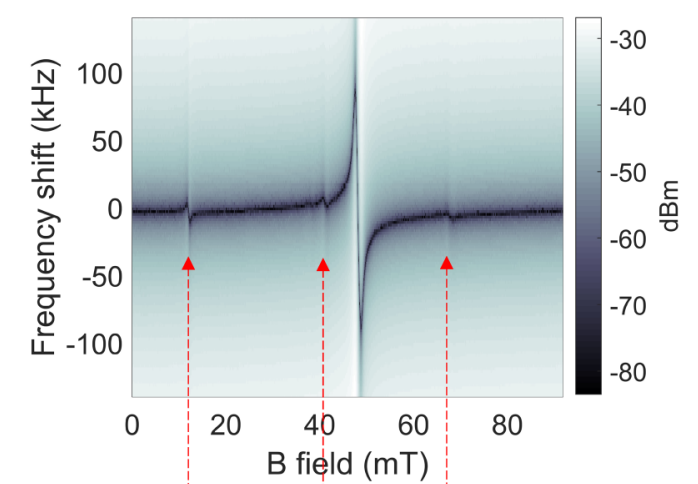

(b)

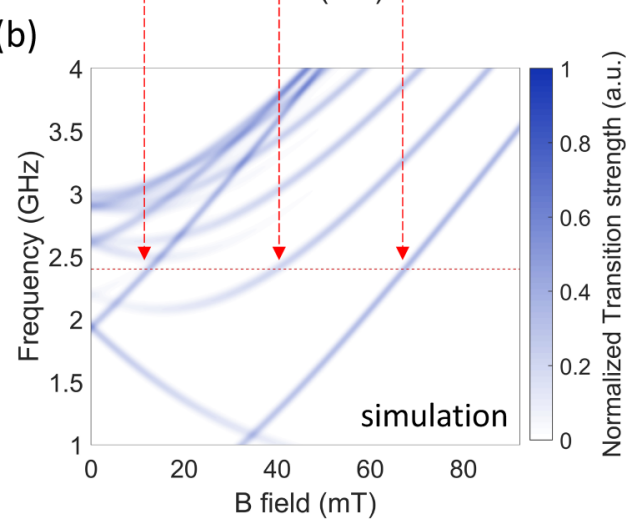

(c)

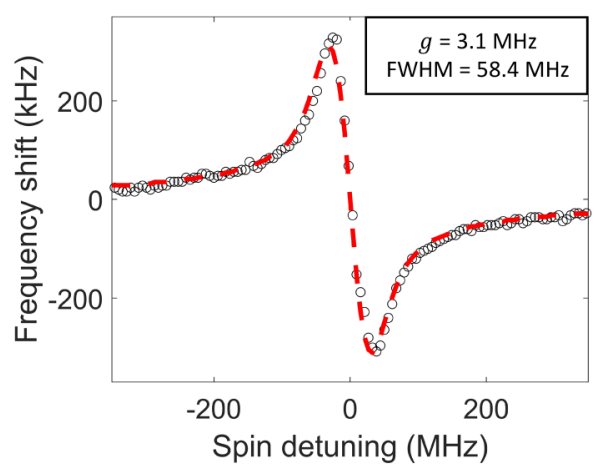

(d)

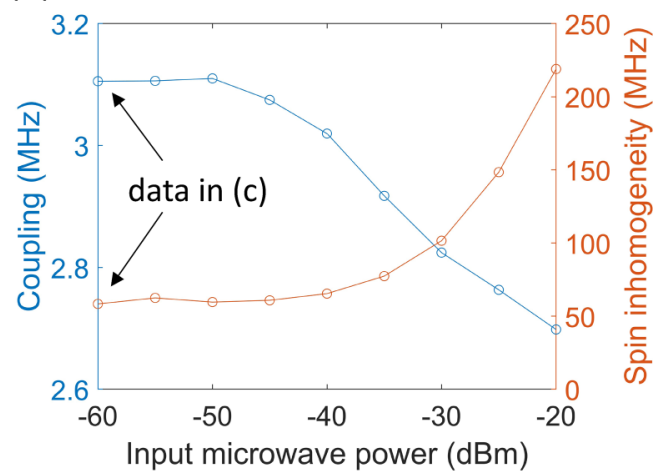

FIG. 6. The simulation and experimental results of the electron paramagnetic resonance (EPR) measurements. (a) A full EPR spectrum taken at $4 \mathrm{~K}$. The dispersive shift of the microwave resonator frequency indicates the coupling between the atomic ensemble and the resonator mode. The coupling at $48 \mathrm{mT}$ was contributed from the even isotope of erbium with zero nuclear spin. The other three couplings located at 12,41 , and $68 \mathrm{mT}$ were from the ${ }^{167}$ Er with $\frac{7}{2}$ nuclear spin. (b) A simulation showing the allowed hyperfine transitions with $\Delta m_{s}= \pm 1$ and $\Delta m_{I}=0$ selection rules under $\mathbf{B}_{\mathrm{AC}} \| a$ condition. The red dashed line indicates the microwave resonator center frequency. The red dashed arrows show the agreement of the $\mathrm{Z}_{1}$ spin hyperfine structure between the experiment and the simulation. (c) A detailed scan of the spin ensemble-cavity coupling centered at $48 \mathrm{mT}$ taken at a temperature of $1 \mathrm{~K}$. Each data point is obtained by a minimum search of the beat note signal at the corresponding magnetic field strength. The red dashed line is the fitting result using the model in Ref. [53] by least square method. (d) The power dependence of EPR results.

Planck constant, and $\mu_{0}$ is the vacuum permeability. From the simulation of the cavity, we predicted $\eta \approx 2 \%$, corresponding to an ensemble coupling $\Omega \approx 2.3 \mathrm{MHz}$ at $1 \mathrm{~K}$. In the weak coupling regime, the maximum dispersive shift is proportional to $\Omega^{2} / \Delta$ [52], where $\Delta$ is the spin inhomogeneity.

To increase our measurement sensitivity for dispersive shifts smaller than the linewidth of the microwave cavity [full width at half maximum $(\mathrm{FWHM})=2.8 \mathrm{MHz}]$, we implemented a FM microwave tone to monitor small changes in the microwave cavity resonance frequency [45]. To determine the spin inhomogeneity, we followed the analysis from Ref. [53], where a cavity with frequency $\omega_{0}$ couples to a distribution of $N$ two-level systems with transition frequencies $\omega_{k}$ and damping rates $\gamma$ with strength $g_{k}$ in the weak excitation regime. The cavity transmission function is given by

$$
\begin{aligned}
t(\omega) & =\frac{\frac{\kappa}{2 i}}{\omega-\omega_{0}+\frac{i \kappa}{2}-W(\omega)}, \\
W(\omega) & =\Omega^{2} \int_{-\infty}^{+\infty} \frac{\rho\left(\omega^{\prime}\right)}{\omega-\omega^{\prime}+\frac{i \gamma}{2}} d \omega^{\prime},
\end{aligned}
$$

where $\kappa$ is the linewidth of the cavity, $W(\omega)$ is the ion-cavity coupling term, $\Omega^{2}=\sum_{k} g_{k}^{2}$ is the total coupling between the cavity and the spins, and $\rho(\omega)=\sum_{k} g_{k}^{2} \delta\left(\omega-\omega_{k}\right) / \Omega^{2}$ is the spectral density distribution. For a Gaussian distribution, $\rho(\omega)=\frac{\sqrt{\ln 2}}{\Delta \sqrt{\pi}} e^{-\left(\omega^{2} \ln 2\right) / \Delta^{2}}$, where $\Delta$ is the spin inhomogeneity.

The transmitted FM microwave field was measured on a microwave power meter at the modulation frequency $\omega_{m}$ :

$$
\begin{aligned}
P_{t}= & \text { DC part }+P_{0} \beta\left\{\operatorname{Re}[\chi(\omega)] \cos \omega_{m} t+\operatorname{Im}[\chi(\omega)] \sin \omega_{m} t\right\} \\
& + \text { terms oscillating at } 2 \omega_{m},
\end{aligned}
$$

where $\beta$ is the modulation strength and $\chi(\omega)=$ $t(\omega) t^{*}\left(\omega+\omega_{m}\right)-t^{*}(\omega) t\left(\omega-\omega_{m}\right)$. When $\omega_{m} \ll \kappa$ and $\kappa \ll \Delta, \chi(\omega)$ has the shape of the derivative of $|t(\omega)|^{2}$, which transforms the resonance peak to a zero-crossing point. We measured the beat-note signal on a spectral analyzer, taking advantage of the large detection dynamic range to maximize our frequency sensitivity. Using this technique, we were able to measure kilohertz frequency shifts that were $\sim 1000$ times smaller than the resonator linewidth.

As shown in Fig. 6(a), as the magnetic field was swept such that the spin frequency crossed the bare cavity frequency, we observed a dispersive response of the microwave resonator frequency. The strongest signal at $48 \mathrm{mT}$ was from the even isotopes, where other coupling points at $\mathbf{B}_{\mathrm{DC}}=12,41$, 
(a)

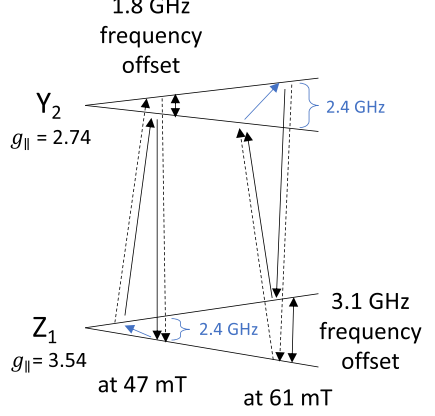

(b)

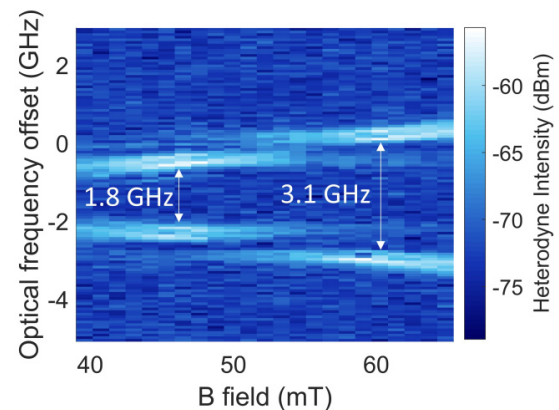

(c)

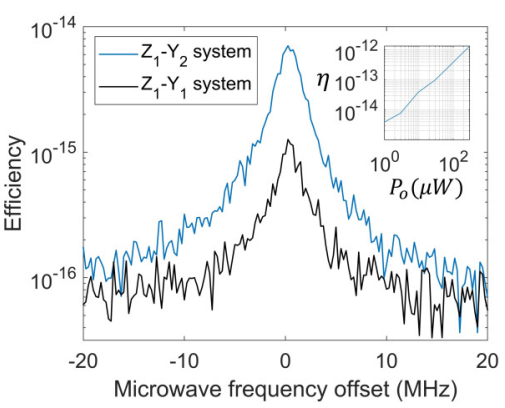

FIG. 7. The microwave-to-optical transduction experiment using Raman heterodyne detection, with the $Z_{1}-Y_{2}$ and $Z_{1}-Y_{1}$ systems. (a) The $\mathrm{Z}_{1}-\mathrm{Y}_{2}$ system used in (b). For example, at $47 \mathrm{mT}$, the $\mathrm{Z}_{1}$ spins are matched to be on resonance with the $2.4 \mathrm{GHz}$ microwave resonator. Two $\Lambda$ systems were usable for transduction with a $1.8 \mathrm{GHz}$ optical frequency offset. (b) The Raman heterodyne spectroscopy using the $\mathrm{Z}_{1}-\mathrm{Y}_{2}$ system. Two signal branches at $47 \mathrm{mT}$ corresponded to two $\Lambda$ systems, and the other two branches at $61 \mathrm{mT}$ corresponded to two $\mathrm{V}$ systems. (c) Transduction efficiency vs input microwave frequency using $\mathrm{Z}_{1}-\mathrm{Y}_{1}$ and $\mathrm{Z}_{1}-\mathrm{Y}_{2}$ systems. The current bandwidth was limited by the loop-gap microwave resonator linewidth $(2.8 \mathrm{MHz})$. The inset figure shows the efficiency $(\eta)$ dependence upon optical pump power $\left(P_{o}\right)$ using the $\mathrm{Z}_{1}-\mathrm{Y}_{2}$ system.

and $68 \mathrm{mT}$ were attributed to resonances with ${ }^{167} \mathrm{Er}$ isotope hyperfine transitions. To understand the hyperfine coupling points, we simulated the $Z_{1}$ level hyperfine structure of ${ }^{167} \mathrm{Er}$ using the parameters presented in Ref. [48]. The calculated hyperfine transitions were showed in Fig. 6(b), where the transition strength was evaluated by taking the inner product of the initial and final states mediated by a $\sigma_{x} \otimes I_{N}$ operator to factor in $\Delta m_{s}= \pm 1$ and $\Delta m_{I}=0$ selection rules for $\mathbf{B}_{\mathrm{AC}} \| a$. There are three allowed hyperfine transitions crossing the $2.4 \mathrm{GHz}$ loop-gap resonance at different bias magnetic fields, which agrees with the experimental data, as indicated by the red dashed arrows.

Figure 6(c) shows the detail of the scan $\sim 48 \mathrm{mT}$ at $1 \mathrm{~K}$, where the even isotope dispersive shift increased compared with Fig. 6(a) due to a larger ground-state spin population difference at the lower temperature. We calculated the $Z_{1}$ electron spin inhomogeneity to be $58.4 \mathrm{MHz}$ with an ensemble coupling of $3.1 \mathrm{MHz}$, assuming a Gaussian line shape for the spin inhomogeneity to fit the data [see Fig. 6(c)]. The small coupling strength observed here is expected due to the small size of the sample used and can be further improved by using a larger crystal to increase the filling factor [54]. To ensure that we were not limited by power broadening or saturation effects on the spin coupling, we swept the input microwave power from -60 to $-20 \mathrm{dBm}$, as shown in Fig. 6(d). We observed that the fitted spin inhomogeneity and ensemble coupling remained roughly constant for the input microwave powers $<-50 \mathrm{dBm}$. However, the fitted $58.4 \mathrm{MHz}$ spin inhomogeneity is still an upper bound, given that we expect some spatial magnetic field inhomogeneity from our homemade magnet over the $3.5 \times 2.5 \times 2 \mathrm{~mm}$ sample volume within the microwave resonator.

\section{M2O transduction}

To generate upconverted optical photons, we input a microwave signal and an optical pump into an $\mathrm{Er}^{3+}: \mathrm{YVO}_{4}$ crystal continuously and measured the optical upconverted signal using heterodyne detection [45]. The key to this resonance-enhanced Raman scattering protocol is to employ a suitable three-level system, where three atomic levels are linked via two allowed optical transitions and one allowed microwave transition. Using the $\mathrm{Er}^{3+}: \mathrm{YVO}_{4} \mathrm{Z}_{1}-\mathrm{Y}_{1}$ or $\mathrm{Z}_{1}-$ $\mathrm{Y}_{2}$ systems in an applied bias magnetic field, there are four possible optical transitions and two possible microwave transitions. From the $\mathrm{Er}^{3+}: \mathrm{YVO}_{4}$ selection rules described in the Background section, both the $\mathrm{Z}_{1}-\mathrm{Y}_{1}$ transition with $\sigma$ polarized excitation or the $Z_{1}-Y_{2}$ transition with $\pi$ polarized excitation are suitable for transduction. For either system, the ground or excited state spins can be tuned onto resonance with the microwave resonator using the bias field to make use of a $\Lambda$ or V system.

To demonstrate $\mathrm{M} 2 \mathrm{O}$ transduction, we used the $\mathrm{Z}_{1}-\mathrm{Y}_{2}$ system under $\pi$ polarization excitation with a $200-\mu$ m-thick sample, driving with a fixed $2.4 \mathrm{GHz} R F$ at a fixed $1 \mathrm{dBm}$ RF power input and $4 \mu \mathrm{W}$ of optical pump power, as shown in Fig. 7. The four configurations that generate the transduction signal [Figs. 7(a) and 7(b)] are apparent. Two of them were centered at $B=47 \mathrm{mT}$, corresponding to the $\mathrm{Z}_{1}$ spins involved in $\Lambda$ systems. These two branches were offset by 1.8 $\mathrm{GHz}$ in optical frequency, which corresponds to the $\mathrm{Y}_{2}$ spin splitting. The other two signals that appear at $B=61 \mathrm{mT}$ were generated from the $V$ systems involving the $Y_{2}$ spin, where the $3.1 \mathrm{GHz}$ optical frequency offset was the $Z_{1}$ spin splitting.

In $\mathrm{Er}^{3+}: \mathrm{YVO}_{4}$, the $\mathrm{Z}_{1}-\mathrm{Y}_{2}$ system is more attractive than $\mathrm{Z}_{1}-\mathrm{Y}_{1}$ because the two involved optical transitions have stronger transition strength (Table I), and the transduction efficiency scales as the product square of the individual dipole moments in the limit of restricted optical pump power. As a result, the $Z_{1}-Y_{2}$ system should be more efficient by a factor of 6. By optimizing the applied magnetic field strength and optical frequency, we measured the transduction signal using the $Z_{1}-Y_{1}$ and $Z_{1}-Y_{2}$ systems and observed that the relative signal strength is consistent with the prediction, as shown in Fig. 7(c). The trend in efficiency vs input microwave frequency followed the loop-gap resonator line shape $(\mathrm{FWHM}=2.8 \mathrm{MHz}$ ) that determines the current transduction bandwidth. The inset in Fig. 7(c) shows the optical pump 
power $\left(P_{o}\right)$ dependence of $\mathrm{Z}_{1}-\mathrm{Y}_{2}$ system transduction efficiency. Up to $300 \mu \mathrm{W}$ input power, limited by the setup, the transduction efficiency increased linearly. Finally, the highest measured efficiency of this initial demonstration of the transduction process is $1.3 \times 10^{-12}$ with $1 \mathrm{dBm}$ microwave and $300 \mu \mathrm{W}$ optical pump power. To further increase the efficiency, an improved microwave resonator with higher $Q$ and a resonant optical cavity are needed, as discussed in Refs. $[18,20]$.

To compare with other rare-earth materials for $\mathrm{M} 2 \mathrm{O}$ transduction applications, we refer to the well-studied $\mathrm{Er}^{3+}: \mathrm{Y}_{2} \mathrm{SiO}_{5}$ crystal in Ref. [14], where a cavity-enhanced M2O transduction experiment using a $10 \mathrm{ppm} \mathrm{Er}^{3+}: \mathrm{Y}_{2} \mathrm{SiO}_{5}$ and all relevant spectroscopic parameters were presented. To evaluate the $\mathrm{M} 2 \mathrm{O}$ transduction process with a threelevel system, we compared the material parameter $\zeta=$ $\left(\frac{d_{31} d_{32} \mu_{21} \rho}{\Delta_{o} \Delta_{\mu}}\right)^{2}$, which scales as the efficiency (in the lowefficiency regime). The value of $\zeta$ for the $\mathrm{Er}^{3+}: \mathrm{YVO}_{4} \mathrm{Z}_{1}-\mathrm{Y}_{2}$ system is $800 \times$ larger than the $\mathrm{Er}^{3+}: \mathrm{Y}_{2} \mathrm{SiO}_{5}$ case due to its relatively narrow optical and spin inhomogeneities at higher concentration and also the stronger dipole moment. However, it has been reported that $\mathrm{Er}^{3+}: \mathrm{Y}_{2} \mathrm{SiO}_{5}$ can also have relatively narrow optical inhomogeneity at higher concentrations $(510 \mathrm{MHz}$ at $200 \mathrm{ppm}$ compared with $170 \mathrm{MHz}$ at $10 \mathrm{ppm}$ ) [28]. Assuming the spin inhomogeneity was unchanged, the $\zeta_{\mathrm{Er}^{3+}: \mathrm{YVO}_{4}} / \zeta_{\mathrm{Er}^{3+}: \mathrm{Y}_{2} \mathrm{SiO}_{5}}$ ratio would be reduced to 18 from 800 if the higher concentration $\mathrm{Er}^{3+}: \mathrm{Y}_{2} \mathrm{SiO}_{5}$ could be used. Therefore, both $\mathrm{Er}^{3+}: \mathrm{Y}_{2} \mathrm{SiO}_{5}$ and $\mathrm{Er}^{3+}: \mathrm{YVO}_{4}$ materials can potentially be improved at higher concentration if they still possess relatively narrow optical and spin inhomogeneities. Finally, we expect that the $58.4 \mathrm{MHz} \mathrm{Er}{ }^{3+}: \mathrm{YVO}_{4}$ spin inhomogeneity measured here was limited by the spatial magnetic field inhomogeneity, which suggests that the above $\zeta_{\mathrm{Er}^{3+}: \mathrm{YVO}_{4}}$ analysis is a lower bound. In conclusion, the relatively narrow ensemble inhomogeneity and strong dipole moment make $\mathrm{Er}^{3+}: \mathrm{YVO}_{4}$ promising for the transduction application.

\section{CONCLUSIONS}

In this paper, we presented the experimental characterization of $\mathrm{Er}^{3+}: \mathrm{YVO}_{4}$ including the electronic Zeeman $g$ tensors of the optically excited states $Y_{1}$ and $Y_{2}$, the optical properties of the telecommunication wavelength transitions $Z_{1}-Y_{1}$ and $Z_{1}-Y_{2}$, the spin inhomogeneity of the $Z_{1}$ spin transition, and an initial demonstration of $\mathrm{M} 2 \mathrm{O}$ transduction using $\mathrm{Er}^{3+}: \mathrm{YVO}_{4}$. The optical inhomogeneity of a $140 \mathrm{ppm}$ sample is exceptionally narrow, and the total oscillator strengths are relatively strong compared with other erbium-doped materials. We also measured an upper bound of $58.4 \mathrm{MHz}$ for the minimum spin inhomogeneity of the microwave transition. Therefore, the highly absorptive optical transitions and the narrow inhomogeneities make $\mathrm{Er}^{3+}: \mathrm{YVO}_{4}$ a promising material for $\mathrm{M} 2 \mathrm{O}$ transduction applications. Next steps toward more efficient $\mathrm{M} 2 \mathrm{O}$ transduction will involve using $\mathrm{Er}^{3+}: \mathrm{YVO}_{4}$ in the cavity-enhanced regime in both optical and spin domains.

\section{ACKNOWLEDGMENTS}

This paper was supported by Army Research Office (ARO/LPS) (CQTS) Grant No. W911NF1810011, Office of Naval Research Award No. N00014-19-1-2182, Air Force Office of Scientific Research Grant No. FA9550-18-1-0374 and No. FA9550-21-1-0055, Northrop Grumman, and Weston Havens Foundation. All work at Montana State University and part of the work at Caltech was supported by the National Science Foundation under Grant No. 1936350 J.G.B. acknowledges the support of the American Australian Association's Northrop Grumman Fellowship. I.C. and J.R. acknowledge support from the Natural Sciences and Engineering Research Council of Canada (Grant No. PGSD2502755-2017 and No. PGSD3-502844-2017). We would also like to acknowledge E. Miyazono for the initial measurements of $\mathrm{Er}^{3+}: \mathrm{YVO}_{4}$ and $\mathrm{M}$. Shaw for help with superconducting photon detectors.
[1] F. Arute et al., Quantum supremacy using a programmable superconducting processor, Nature 574, 505 (2019).

[2] C. D. Bruzewicz, J. Chiaverini, R. McConnell, and J. M. Sage, Trapped-ion quantum computing: progress and challenges, Appl. Phys. Rev 6, 021314 (2019).

[3] J. Yin et al., Satellite-based entanglement distribution over 1200 kilometers, Science 356, 1140 (2017).

[4] M. Pompili, S. L. N. Hermans, S. Baier, H. K. C. Beukers, P. C. Humphreys, R. N. Schouten, R. F. L. Vermeulen, M. J. Tiggelman, L. dos Santos Martins, B. Dirkse, S. Wehner, and R. Hanson, Realization of a multi-node quantum network of remote solid-state qubits, Science 372, 259 (2021).

[5] K. Heshami, D. G. England, P. C. Humphreys, P. J. Bustard, V. M. Acosta, J. Nunn, and B. J. Sussman, Quantum memories: emerging applications and recent advances, J. Mod. Opt. 63, 2005 (2016).

[6] Y. Yu, F. Ma, X.-Y. Luo, B. Jing, P.-F. Sun, R.-Z. Fang, C.W. Yang, H. Liu, M.-Y. Zheng, X.-P. Xie, W.-J. Zhang, L.-X. You, Z. Wang, T.-Y. Chen, Q. Zhang, X.-H. Bao, and J.-W. Pan,
Entanglement of two quantum memories via fibres over dozens of kilometres, Nature 578, 240 (2020).

[7] S. Kumar, N. Lauk, and C. Simon, Towards long-distance quantum networks with superconducting processors and optical links, Quantum Sci. Technol. 4, 045003 (2019).

[8] N. Lauk, N. Sinclair, S. Barzanjeh, J. P. Covey, M. Saffman, M. Spiropulu, and C. Simon, Perspectives on quantum transduction, Quantum Sci. Technol. 5, 020501 (2020).

[9] N. J. Lambert, A. Rueda, F. Sedlmeir, and H. G. L. Schwefel, Coherent conversion between microwave and optical photonsan overview of physical implementations, Adv. Quantum Technol. 3, 1900077 (2020).

[10] A. P. Higginbotham, P. S. Burns, M. D. Urmey, R. W. Peterson, N. S. Kampel, B. M. Brubaker, G. Smith, K. W. Lehnert, and C. A. Regal, Harnessing electro-optic correlations in an efficient mechanical converter, Nat. Phys. 14, 1038 (2018).

[11] M. Mirhosseini, A. Sipahigil, M. Kalaee, and O. Painter, Superconducting qubit to optical photon transduction, Nature $\mathbf{5 8 8}$, 599 (2020). 
[12] A. Rueda, F. Sedlmeir, M. C. Collodo, U. Vogl, B. Stiller, G. Schunk, D. V. Strekalov, C. Marquardt, J. M. Fink, O. Painter, G. Leuchs, and H. G. L. Schwefel, Efficient microwave to optical photon conversion: an electro-optical realization, Optica 3, 597 (2016).

[13] L. Fan, C.-L. Zou, R. Cheng, X. Guo, X. Han, Z. Gong, S. Wang, and H. X. Tang, Superconducting cavity electrooptics: a platform for coherent photon conversion between superconducting and photonic circuits, Sci. Adv. 4, eaar4994 (2018).

[14] X. Fernandez-Gonzalvo, S. P. Horvath, Y.-H. Chen, and J. J. Longdell, Cavity-enhanced Raman heterodyne spectroscopy in $\mathrm{Er}^{3+}: \mathrm{Y}_{2} \mathrm{SiO}_{5}$ for microwave to optical signal conversion, Phys. Rev. A 100, 033807 (2019).

[15] J. Han, T. Vogt, C. Gross, D. Jaksch, M. Kiffner, and W. Li, Coherent Microwave-to-Optical Conversion via Six-Wave Mixing in Rydberg Atoms, Phys. Rev. Lett. 120, 093201 (2018).

[16] R. Hisatomi, A. Osada, Y. Tabuchi, T. Ishikawa, A. Noguchi, R. Yamazaki, K. Usami, and Y. Nakamura, Bidirectional conversion between microwave and light via ferromagnetic magnons, Phys. Rev. B 93, 174427 (2016).

[17] S. Das, V. E. Elfving, S. Faez, and A. S. Sørensen, Interfacing Superconducting Qubits and Single Optical Photons Using Molecules in Waveguides, Phys. Rev. Lett. 118, 140501 (2017).

[18] L. A. Williamson, Y.-H. Chen, and J. J. Longdell, MagnetoOptic Modulator with Unit Quantum Efficiency, Phys. Rev. Lett. 113, 203601 (2014).

[19] C. O'Brien, N. Lauk, S. Blum, G. Morigi, and M. Fleischhauer, Interfacing Superconducting Qubits and Telecom Photons via a Rare-Earth-Doped Crystal, Phys. Rev. Lett. 113, 063603 (2014).

[20] J. G. Bartholomew, J. Rochman, T. Xie, J. M. Kindem, A. Ruskuc, I. Craiciu, M. Lei, and A. Faraon, On-chip coherent microwave-to-optical transduction mediated by ytterbium in $\mathrm{YVO}_{4}$, Nat. Commun. 11, 3266 (2020).

[21] J. R. Everts, M. C. Berrington, R. L. Ahlefeldt, and J. J. Longdell, Microwave to optical photon conversion via fully concentrated rare-earth-ion crystals, Phys. Rev. A 99, 063830 (2019).

[22] C. W. Thiel, Thomas Böttger, and R. L. Cone, Rare-earth-doped materials for applications in quantum information storage and signal processing, J. Lumin. 131, 353 (2011).

[23] J. M. Kindem, J. G. Bartholomew, P. J. T. Woodburn, T. Zhong, I. Craiciu, R. L. Cone, C. W. Thiel, and A. Faraon, Characterization of ${ }^{171} \mathrm{Yb}^{3+}: \mathrm{YVO}_{4}$ for photonic quantum technologies, Phys. Rev. B 98, 024404 (2018).

[24] M. Zhong, M. P. Hedges, R. L. Ahlefeldt, J. G. Bartholomew, S. E. Beavan, S. M. Wittig, J. J. Longdell, and M. J. Sellars, Optically addressable nuclear spins in a solid with a six-hour coherence time, Nature 517, 177 (2015).

[25] Guokui Liu and Bernard Jacquier (eds.), Spectroscopic Properties of Rare Earths in Optical Materials (Springer, Berlin and Tsinghua University Press, 2005), Vol. 83.

[26] J. V. Rakonjac, Y.-H. Chen, S. P. Horvath, and J. J. Longdell, Long spin coherence times in the ground state and in an optically excited state of ${ }^{167} \mathrm{Er}^{3+}: \mathrm{Y}_{2} \mathrm{SiO}_{5}$ at zero magnetic field, Phys. Rev. B 101, 184430 (2020).

[27] Y. Sun, T. Böttger, C. W. Thiel, and R. L. Cone, Magnetic $g$ tensors for the ${ }^{4} \mathrm{I}_{15 / 2}$ and ${ }^{4} \mathrm{I}_{13 / 2}$ states of $\mathrm{Er}^{3+}: \mathrm{Y}_{2} \mathrm{SiO}_{5}$, Phys. Rev. B 77, 085124 (2008).
[28] T. Böttger, Y. Sun, C. W. Thiel, and R. L. Cone, Spectroscopy and dynamics of $\mathrm{Er}^{3+}: \mathrm{Y}_{2} \mathrm{SiO}_{5}$ at $1.5 \mu \mathrm{m}$, Phys. Rev. B 74, 075107 (2006).

[29] M. Rančić, M. P. Hedges, R. L. Ahlefeldt, and M. J. Sellars, Coherence time of over a second in a telecom-compatible quantum memory storage material, Nat. Phys. 14, 50 (2018).

[30] M. N. Popova, S. A. Klimin, S. A. Moiseev, K. I. Gerasimov, M. M. Minnegaliev, E. I. Baibekov, G. S. Shakurov, M. Bettinelli, and M. C. Chou, Crystal field and hyperfine structure of ${ }^{167} \mathrm{Er}^{3+}$ in $\mathrm{YPO}_{4}$ :Er single crystals: high-resolution optical and EPR spectroscopy, Phys. Rev. B 99, 235151 (2019).

[31] R. Fukumori, Y. Huang, J. Yang, H. Zhang, and T. Zhong, Subkilohertz optical homogeneous linewidth and dephasing mechanisms in $\mathrm{Er}^{3+}: \mathrm{Y}_{2} \mathrm{O}_{3}$ ceramics, Phys. Rev. B 101, 214202 (2020).

[32] R. Marino, I. Lorgeré, O. Guillot-Noël, H. Vezin, A. Toncelli, M. Tonelli, J.-L. Le Gouët, and P. Goldner, Energy level structure and optical dephasing under magnetic field in $\mathrm{Er}^{3+}: \mathrm{LiYF}_{4}$ at $1.5 \mu \mathrm{m}$, J. Lumin. 169, 478 (2016).

[33] X. Jiang, D. Pak, A. Nandi, Y. Xuan, and M. Hosseini, Rare earth-implanted lithium niobate: properties and on-chip integration, Appl. Phys. Lett. 115, 071104 (2019).

[34] C. M. Phenicie, P. Stevenson, S. Welinski, B. C. Rose, A. T. Asfaw, R. J. Cava, S. A. Lyon, N. P. de Leon, and J. D. Thompson, Narrow optical line widths in erbium implanted in $\mathrm{TiO}_{2}$, Nano Lett. 19, 8928 (2019).

[35] J. A. Capobianco, P. Kabro, F. S. Ermeneux, R. Moncorge, M. Bettinelli, and E. Cavalli, Optical spectroscopy, fluorescence dynamics and crystal-field analysis of $\mathrm{Er}^{3+}$ in $\mathrm{YVO}_{4}$, Chem. Phys. 214, 329 (1997).

[36] N. Ter-Gabrielyan, V. Fromzel, W. Ryba-Romanowski, T. Lukasiewicz, and M. Dubinskii, Spectroscopic and laser properties of resonantly (in-band) pumped Er: $\mathrm{YVO}_{4}$ and Er:GdVO crystals: a comparative study, Opt. Mater. Express 2, 1040 (2012).

[37] P. Le Boulanger, J.-L. Doualan, S. Girard, J. Margerie, and R. Moncorgé, Excited-state absorption spectroscopy of $\mathrm{Er}^{3+}$ doped $\mathrm{Y}_{3} \mathrm{Al}_{5} \mathrm{O}_{12}, \mathrm{YVO}_{4}$, and phosphate glass, Phys. Rev. B 60, 11380 (1999).

[38] S. Golab, G. Dominiak-Dzik, P. Solarz, T. Lukasiewicz, M. Swirkowicz, I. Sokolska, and W. Ryba-Romanowski, Relaxation dynamics of excited states of $\mathrm{Er}^{3+}$ in $\mathrm{YVO}_{4}$ single crystals, Proc. SPIE 4412, International Conference on Solid State Crystals 2000: Growth, Characterization, and Applications of Single Crystals (SPIE, Zakopane, 2001).

[39] P.-Y. Li, J.-Y. Huang, T.-X. Zhu, C. Liu, D.-C. Liu, Z.-Q. Zhou, C.-F. Li, and G.-C. Guo, Optical spectroscopy and coherent dynamics of ${ }^{167} \mathrm{Er}^{3+}: \mathrm{YVO}_{4}$ at $1.5 \mu \mathrm{m}$ below $1 \mathrm{~K}$, J. Lumin. 225, 117344 (2020).

[40] B. C. Chakoumakos, M. M. Abraham, and L. A. Boatner, Crystal structure refinements of zircon-type $M \mathrm{VO}_{4}(M=\mathrm{Sc}, \mathrm{Y}, \mathrm{Ce}$, Pr, Nd, Tb, Ho, Er, Tm, Yb, Lu), J. Solid State Chem. 109, 197 (1994).

[41] A. Abragam and B. Bleaney, Electron Paramagnetic Resonance of Transition Ions (OUP, Oxford, 2012).

[42] G. F. Koster, J. O. Dimmock, and R. G. Wheeler, Properties of the Thirty-Two Point Groups (MIT Press, Cambridge, MA, 1963), Vol. 24.

[43] Z. Luo and Y. Huang, Physics of Solid-State Laser Materials (Springer Series in Materials Science, 2020), Vol. 289. 
[44] S. Hufner (ed.), Optical Spectra of Transparent Rare Earth Compounds (Academic Press, New York, 1978).

[45] X. Fernandez-Gonzalvo, Y.-H. Chen, C. Yin, S. Rogge, and J. J. Longdell, Coherent frequency up-conversion of microwaves to the optical telecommunications band in an Er:YSO crystal, Phys. Rev. A 92, 062313 (2015).

[46] H.-S. Shi, G. Zhang, and H.-Y. Shen, Measurement of principal refractive indices and the thermal refractive index coefficients of yttrium vanadate, J. Synth. Cryst. 30, 85 (2001).

[47] C. M. Dodson and R. Zia, Magnetic dipole and electric quadrupole transitions in the trivalent lanthanide series: calculated emission rates and oscillator strengths, Phys. Rev. B 86, 125102 (2012).

[48] U. Ranon, Paramagnetic resonance of $\mathrm{Nd}^{3+}, \mathrm{Dy}^{3}+, \mathrm{Er}^{3+}$ and $\mathrm{Yb}^{3+}$ in $\mathrm{YVO}_{4}$, Phys. Lett. A 28, 228 (1968).

[49] L. Veissier, C. W. Thiel, T. Lutz, P. E. Barclay, W. Tittel, and R. L. Cone, Quadratic Zeeman effect and spin-lattice relaxation of $\mathrm{Tm}^{3+}$ :YAG at high magnetic fields, Phys. Rev. B 94, 205133 (2016).

[50] D. S. Sumida and T. Y. Fan, Effect of radiation trapping on fluorescence lifetime and emission cross section mea- surements in solid-state laser media, Opt. Lett. 19, 1343 (1994).

[51] F. S. Ermeneux, R. Moncorge, P. Kabro, J. A. Capobianco, M. Bettinelli, and E. Cavalli, Crystal growth and luminescence properties of $\mathrm{Er}^{3+}$ doped $\mathrm{YVO}_{4}$ single crystals, in Advanced Solid State Lasers, edited by S. Payne and C. Pollack (Optical Society of America, San Francisco, 1996), Vol. 1, paper SM9.

[52] G. Dold, C. W. Zollitsch, J. O'Sullivan, S. Welinski, A. Ferrier, P. Goldner, S. E. de Graaf, T. Lindström, and J. J. L. Morton, High-cooperativity coupling of a rare-earth spin ensemble to a superconducting resonator using yttrium orthosilicate as a substrate, Phys. Rev. Appl. 11, 054082 (2019).

[53] I. Diniz, S. Portolan, R. Ferreira, J. M. Gérard, P. Bertet, and A. Auffèves, Strongly coupling a cavity to inhomogeneous ensembles of emitters: potential for long-lived solid-state quantum memories, Phys. Rev. A 84, 063810 (2011).

[54] G. G. G. King, P. S. Barnett, J. G. Bartholomew, A. Faraon, and J. J. Longdell, Probing strong coupling between a microwave cavity and a spin ensemble with Raman heterodyne spectroscopy, Phys. Rev. B 103, 214305 (2021). 